\title{
Conflict between place and response navigation strategies: Effects on vicarious trial and error (VTE) behaviors
}

\author{
Brandy Schmidt, ${ }^{1,2}$ Andrew Papale, ${ }^{2}$ A. David Redish, ${ }^{2,3}$ and Etan J. Markus ${ }^{1,3}$ \\ ${ }^{1}$ Department of Psychology, University of Connecticut, Storrs, Connecticut 06269, USA; ${ }^{2}$ Department of Neuroscience, \\ University of Minnesota, Minneapolis, Minnesota 55455, USA
}

\begin{abstract}
Navigation can be accomplished through multiple decision-making strategies, using different information-processing computations. A well-studied dichotomy in these decision-making strategies compares hippocampal-dependent "place" and dorsal-lateral striatal-dependent "response" strategies. A place strategy depends on the ability to flexibly respond to environmental cues, while a response strategy depends on the ability to quickly recognize and react to situations with welllearned action-outcome relationships. When rats reach decision points, they sometimes pause and orient toward the potential routes of travel, a process termed vicarious trial and error (VTE). VTE co-occurs with neurophysiological information processing, including sweeps of representation ahead of the animal in the hippocampus and transient representations of reward in the ventral striatum and orbitofrontal cortex. To examine the relationship between VTE and the place/response strategy dichotomy, we analyzed data in which rats were cued to switch between place and response strategies on a plus maze. The configuration of the maze allowed for place and response strategies to work competitively or cooperatively. Animals showed increased VTE on trials entailing competition between navigational systems, linking VTE with deliberative decision-making. Even in a well-learned task, VTE was preferentially exhibited when a spatial selection was required, further linking VTE behavior with decision-making associated with hippocampal processing.
\end{abstract}

When deciding what, how, and when to do something, different decision-making strategies are available. There is the computationally slow, but flexible, deliberative system and the computationally fast, but inflexible, habit system (for review, see van der Meer et al. 2012). In humans, deliberative decision-making encompasses several steps: exploring and recognizing the environment, imagining and predicting possible routes, evaluating their respective outcomes, and finally making the choice to take action. Similarly, when rats reach a choice point, they frequently pause and turn serially toward their optional routes of travel, a process termed vicarious trial and error (VTE) (Muenzinger and Gentry 1931; Muenzinger 1938; Tolman 1938). VTE could behaviorally reflect deliberative decision-making as the rat resolves the conflict between outcomes. Neurophysiological studies support the hypothesis that during VTE an animal is using its "cognitive map" by internally representing potential pathways (Johnson and Redish 2007) and evaluating future outcomes (van der Meer and Redish 2010). Various studies have shown that VTE is supported by the hippocampus (Hu and Amsel 1995; Voss et al. 2011), occurs early in learning (Tolman 1939; van der Meer and Redish 2010), and increases with changes in task demands (Blumenthal et al. 2011). However, to date, it has been difficult to determine the degree to which VTE behavior is linked to the different decisionmaking systems available.

Spatial tasks can be accomplished by multiple decisionmaking strategies, using different information-processing computations. The current study utilized the dichotomy between hippocampal-dependent "place" and dorsal-lateral striatal-dependent "response" systems (O'Keefe and Nadel 1978; Packard and

\footnotetext{
${ }^{3}$ Corresponding authors

E-mail redish@umn.edu

E-mail etan.markus@uconn.edu

Article is online at http://www.learnmem.org/cgi/doi/10.1101//m.028753.112.
}

McGaugh 1996; Redish 1999). Importantly, the two systems can compensate for one another during decision-making, one taking over if the other is impaired (Packard and McGaugh 1996; Packard 1999).

These two navigational systems also compete with each other during decision-making (Schmidt et al. 2009; Jacobson et al. 2012). Rats trained to interchangeably use both place and response strategies on a modified plus maze made more errors on trials that required place and response strategies to work competitively (both strategies indicated different goal arms) than cooperatively (both strategies indicated the same goal arm). Even when trained at asymptotic performance, when errors were made, they were predominantly made on competitive trials. Further supporting the role of competition between these systems, errors made on competitive trials were the result of the rat using the alternative navigation strategy.

We measured VTE on three tasks where place and response strategies competed against each other (Schmidt et al. 2009; Jacobson et al. 2012). In the Fixed Place and Response Task ("Fixed Task") rats were trained to continuously switch between a strategy defined by place (e.g., go to the east arm) and a strategy defined by a response (e.g., make a right-hand turn). A flashing light cued the place trial, but did not indicate the goal arm. Place and response trials were interwoven with no more than three consecutive trials of one trial type. In the Novel Place and Fixed Response Task ("Novel Place Task") rats had to learn a new place arm daily during the place trials and continue to make a right-hand turn during the response trials. In the Novel Response and Fixed Place Task ("Novel Response Task") rats were trained to go to the original place arm (east arm) during the place trials and learned to make a novel response (left-hand turn) during the response trials. Testing the rats on the three different tasks, one in which both place and response strategies were overtrained, and comparing these to tasks that introduce 
hippocampal-dependent learning and nonhippocampal-dependent learning allowed us to determine the effects of learning on VTE. Additionally, comparing VTE between competitive and cooperative trials across the three tasks provided a unique opportunity to dissociate the effects of the task demands from learning. VTE was quantitatively measured as the integrated absolute angular velocity (IdPhi) across the pass through the central choicepoint (Papale et al. 2012).

\section{Results}

We were able to replicate Schmidt et al. (2009) and Jacobson et al. (2012) and train rats to continuously switch between place and response strategies (within a session) on the Fixed Task, Novel Place Task, and Novel Response Task (Fig. 1; see Materials and Methods). As a rat reaches a choice point it frequently pauses and orients toward potential routes of travel, a process termed vicarious trial and error (VTE) (Muenzinger and Gentry 1931; Muenzinger 1938; Tolman 1938). VTE behavior was quantified by the IdPhi measure, the integrated absolute change in angular displacement of the rat's head (Papale et al. 2012; see Materials and Methods). Theoretically, the IdPhi distribution should be positively skewed (Fig. 2A), the positively skewed "bump" resulting from a high proportion of large IdPhis resulting from purported VTE behaviors. In the current study, rats exhibited both non-VTE and VTE behavior at the choice point (Fig. 2B-E). Unexpectedly, the IdPhi distribution in the analysis also revealed a shallow negative tail (Fig. 3A,
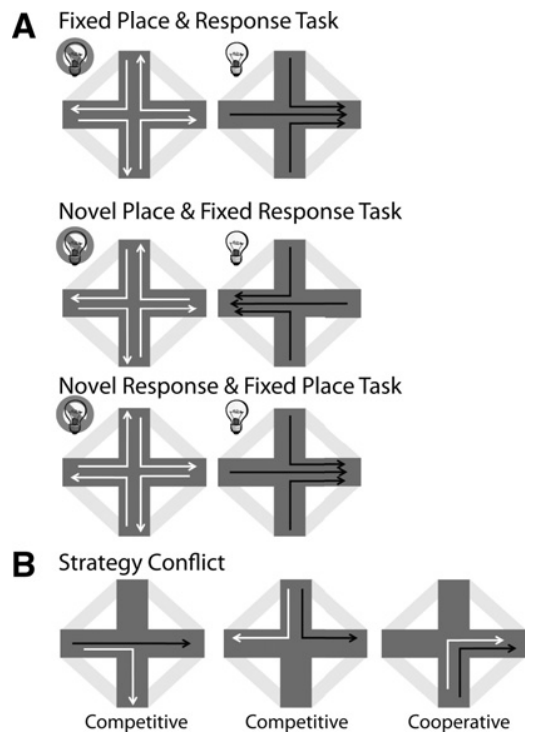

Figure 1. (A) On the Fixed Task (top), rats were trained to switch between a response ("right-hand turn," white arrows) and a place ("east arm," black arrows) navigation strategy. Place trials were cued with a flashing light (the light did not indicate which was the correct goal arm, only that it was a place trial). Place and response trials were interwoven with no more than three consecutive trials of one trial type. On the Novel Place Task (middle) the rats were trained to go to a pseudo-randomly assigned place goal arm for the day (north, south east, west), while continuing to make a right-hand turn on response trials. On the Novel Response Task (bottom) the rats learned to make a lefthand turn, while continuing to go to the original place arm (east arm). (B) Different start arms permitted the navigation strategies to work in conflict or synergistically to reach the goal arm. On competitive trials, place and response strategies indicated different goal locations (left and middle). On cooperative trials, both place and response strategies indicated the same goal location (right).
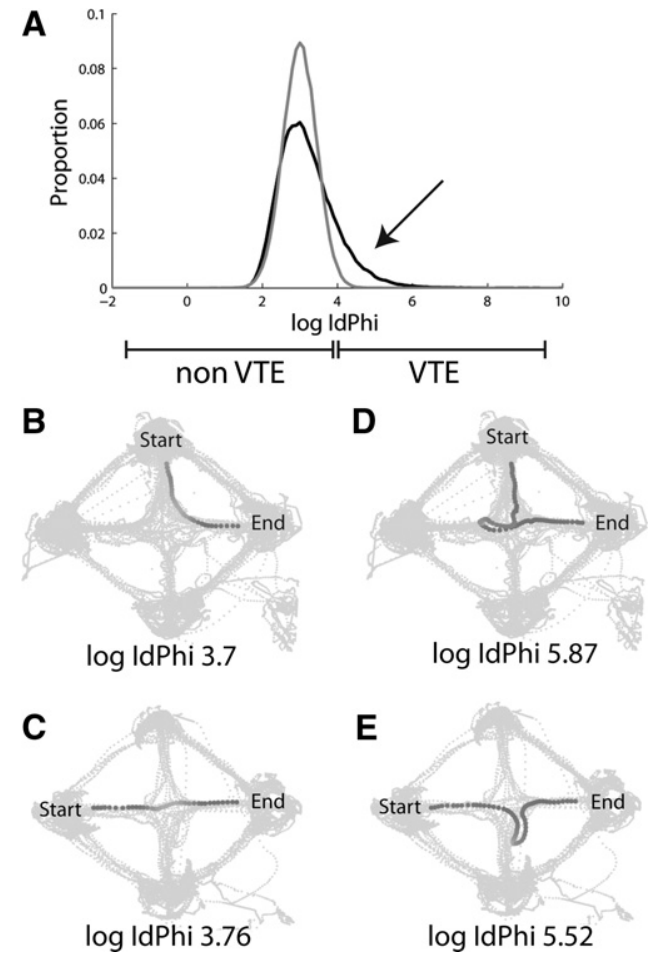

Figure 2. (A) Schematic of IdPhi distribution. VTE behavior is measured by the angular displacement of the rat's head (IdPhi). The greater the displacement, the greater the IdPhi score, as seen during VTE behavior. Note that the presence of VTE trials would produce a positively skewed tail due to the increased IdPhis on VTE trials (black), as compared to a normal distribution (gray). $(B, C)$ Examples of non-VTE laps. $(D, E)$ Examples of VTE laps.

black arrow). When we plotted each traversal (left, right, straight), we found that the source of the left tail resulted from the straight trajectories. Instead of a positively skewed distribution, straight trajectories had a more platykurdic distribution resulting from the naturally lower IdPhi of a regular straight trajectory and the high IdPhi of VTE trials. This does not imply that VTE is not exhibited on straight trials. In fact, examining across the trajectory (straight, left, right) revealed that there is a high proportion of VTE on straight trajectory traversals as well (Fig. 3B). In addition to our IdPhi measurements of VTE, we analyzed differences in reaction time (i.e., start of the trial to entering goal arm) across tasks. As expected, reaction time and IdPhi were positively correlated $(r=0.89, P<0.0001)$.

\section{Vicarious trial and error: Hippocampal vs. nonhippocampal learning}

Previous studies have shown that VTE is impaired in hippocampal amnesic humans (Voss et al. 2011) and in rodents with hippocampal lesions (Hu and Amsel 1995), and is correlated with hippocampal activity (Hu et al. 2006; Johnson and Redish 2007; Voss et al. 2011). In the current study, VTE behaviors were seen on both hippocampal and nonhippocampal tasks: the Fixed Task, Novel Place Task, and Novel Response Task. The IdPhi distribution showed a positively skewed "bump" resulting from a large proportion of VTE behaviors (Fig. 3, gray arrow). Though VTE behavior was exhibited on all tasks (Fig. 3), it was greatest on the Novel Place Task (Novel Place vs. Fixed: $\chi^{2}=68.1, P<0.001$; Novel Place vs. Novel Response: $\chi^{2}=113.5, P<0.001$ ) (Fig. 3C). 
A

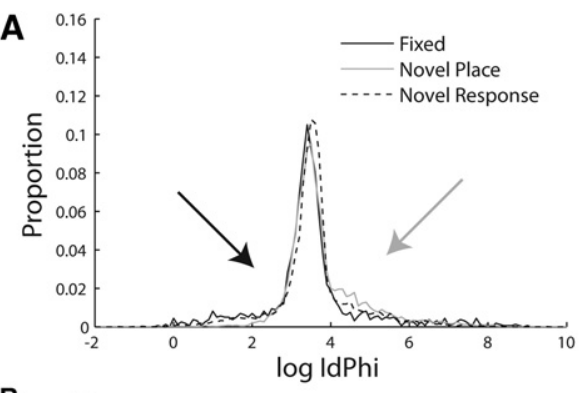

B
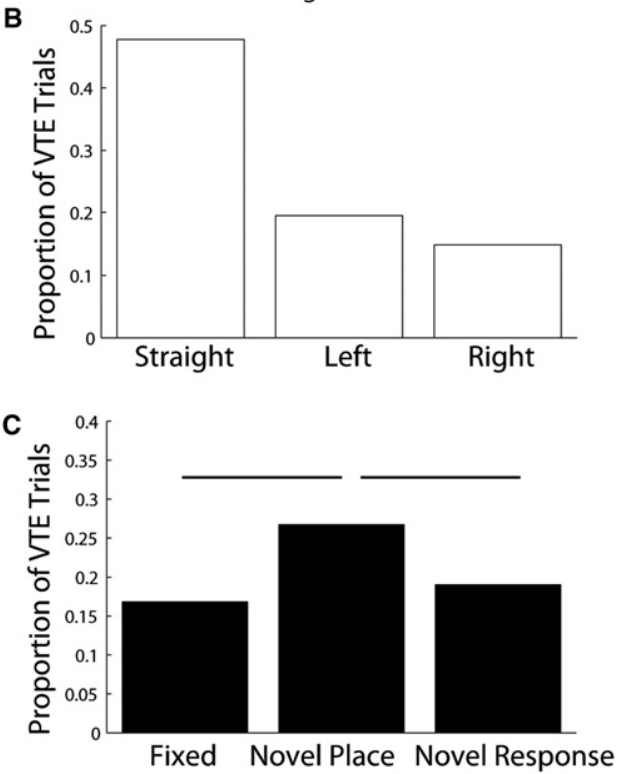

Figure 3. (A) VTE was measured on three tasks: Fixed Task (black line), Novel Place Task (gray line), and Novel Response Task (dashed line). Note the positively skewed bump (gray arrow) resulting from a large number of VTE events. Theoretically, the VTE distribution is positively skewed (cf. Fig. $2 \mathrm{~A}$ ); however, the data revealed a shallow negatively skewed tail (black arrow). Closer examination revealed that these tails resulted from straight trajectory traversals. Straight trajectory traversals may "naturally" have a lower IdPhi. (B) This does not imply that VTE is not exhibited on straight trials. In fact, examining across the trajectory (straight, left, right) revealed that there was a high proportion of VTE on straight trajectory traversals. (C) Examining VTE between the three tasks revealed that VTE was greatest on the Novel Place Task. (Black horizontal line) $P<0.01$.

\section{Vicarious trial and error: Place trials vs. response trials}

We compared the proportion of VTE on place trials to that on response trials. Correct performance on place trials is hippocampal-dependent while correct response trial performance is hippocampal-independent (Jacobson et al. 2012). We predicted that VTE would be greater on trials where the animal was required to use a hippocampal-dependent place strategy. The VTE distribution on place and response trials is seen in Figure 4 . A $2 \times 3$ ANOVA on the Rank-transformed IdPhi found a main effect of navigation strategy (place/response) $\left(F_{(1,14117)}=\right.$ 27.4, $P<0.0001)$ and task (Fixed/Novel Place/Novel Response) $\left(F_{(2,14117)}=37.4, P<0.0001\right)$, and a navigation strategy $\times$ task interaction $\left(F_{(2,14117)}=232.1, P<0.0001\right)$.

Collapsing place and response trials across the three tasks revealed that VTE was observed more during place trials than during response trials $\left(\chi^{2}=168.0, P<0.0001\right)$ (Fig. 5A). However, different patterns of VTE were seen across tasks. We observed more VTE when the rat was cued to use a place strategy on the overtrained Fixed Task $\left(\chi^{2}=16.9, P<0.0001\right)$ (Fig. 5B). Similarly, VTE was greater on place-cued trials in rats trained to learn a novel daily place arm (Novel Place Task; $\chi^{2}=266.3, P<0.0001$ ) (Fig. 5C). Next, we examined whether VTE would persist on place trials while the rat learned a hippocampal-independent novel response (left-hand turn) (Jacobson et al. 2012) in the Novel Response Task. The differences in VTE between place and response strategy cued trials on the Novel Response Task were not significant after correcting for multiple comparisons $\left(\chi^{2}=5.9, P=0.015\right)$ (Fig. $5 \mathrm{D})$. Similar to VTE, reaction time was greater on place trials (Kruskal-Wallis; $\chi^{2}=99.66, P<0.0001$ ).

\section{Vicarious trial and error: Strategy conflict/competition}

VTE has been demonstrated on several spatial decision-making behavioral paradigms where rats had to choose between multiple options for food reward. VTE was preferentially seen at the highcost choice point on the Multiple-T-Maze task (Johnson and Redish 2007; van der Meer and Redish 2010; Blumenthal et al. 2011). VTE was seen at the choice point on a spatial delay discounting task, a task where rats had to choose between a smaller immediate reward and a larger delayed reward (Papale et al. 2012). Theorists posit that VTE occurs in behavioral paradigms with changing task demands (Johnson et al. 2012).

To expand on the role of VTE in deliberative decisionmaking, we examined VTE on trials where rats had to choose between two different navigation strategies. VTE was examined on "competitive strategy" trials (place and response strategies indicated different goal arms) and "cooperative strategy" trials (place and response strategies indicated the same goal arm). A $2 \times 3$ ANOVA on the Rank-transformed IdPhi found a main effect of strategy conflict (competitive/cooperative) $\left(F_{(1,14117)}=45.3, P<\right.$ $0.0001)$ and task $\left(F_{(2,14117)}=34.6, P<0.0001\right)$, and a strategy conflict $\times$ task interaction $\left(F_{(2,14117)}=76.1, P<0.0001\right)$.

Collapsing competitive and cooperative trials across tasks revealed that VTE was greater on competitive trials $\left(\chi^{2}=311.2\right.$, $P<0.0001$ ) (Fig. 6A). Further examination revealed similar patterns across tasks. Though not significantly different when corrected for multiple comparisons, VTE was also exhibited more on competitive than on cooperative trials on the Fixed Task $\left(\chi^{2}=5.3, P=0.022\right.$ ) (Fig. 6B). The lack of significance could be explained by the fact that both navigation strategies were well learned in this familiar task. We predicted that VTE would be observed when a new contingency was introduced in a familiar environment. Therefore, we then investigated whether strategy conflict would modulate VTE on the Novel Place Task and Novel Response Task, tasks with new learning. As predicted, VTE was exhibited more on competitive trials on the Novel Place Task $\left(\chi^{2}=\right.$ 197.5, $P<0.0001$ ) (Fig. 6C) and on the Novel Response Task $\left(\chi^{2}=106.8, P<0.0001\right)$ (Fig. 6D). Consistent with the VTE results, reaction time was greater on competitive trials (KruskalWallis; $\left.\chi^{2}=120.72, P<0.0001\right)$.

\section{Vicarious trial and error: Trial accuracy}

Previous studies have shown that VTE occurs early in learning (Tolman 1938; van der Meer and Redish 2010). Lesion and pharmacological studies suggest a relationship between VTE and performance; manipulations that impair performance also show decreased VTE behavior (Hu and Amsel 1995; Blumenthal et al. 2011). Therefore, we examined whether VTE was modulated by performance accuracy. The $2 \times 3$ ANOVA on the Rank-transformed IdPhi found a main effect of trial accuracy (correct/error) $\left(F_{(1,14117)}=160.8, P<0.0001\right)$ and task $\left(F_{(2,14117)}=12.9, P<\right.$ $0.0001)$, and a trial accuracy $\times$ task interaction $\left(F_{(2,14117)}=10.4\right.$, $P<0.0001)$. 


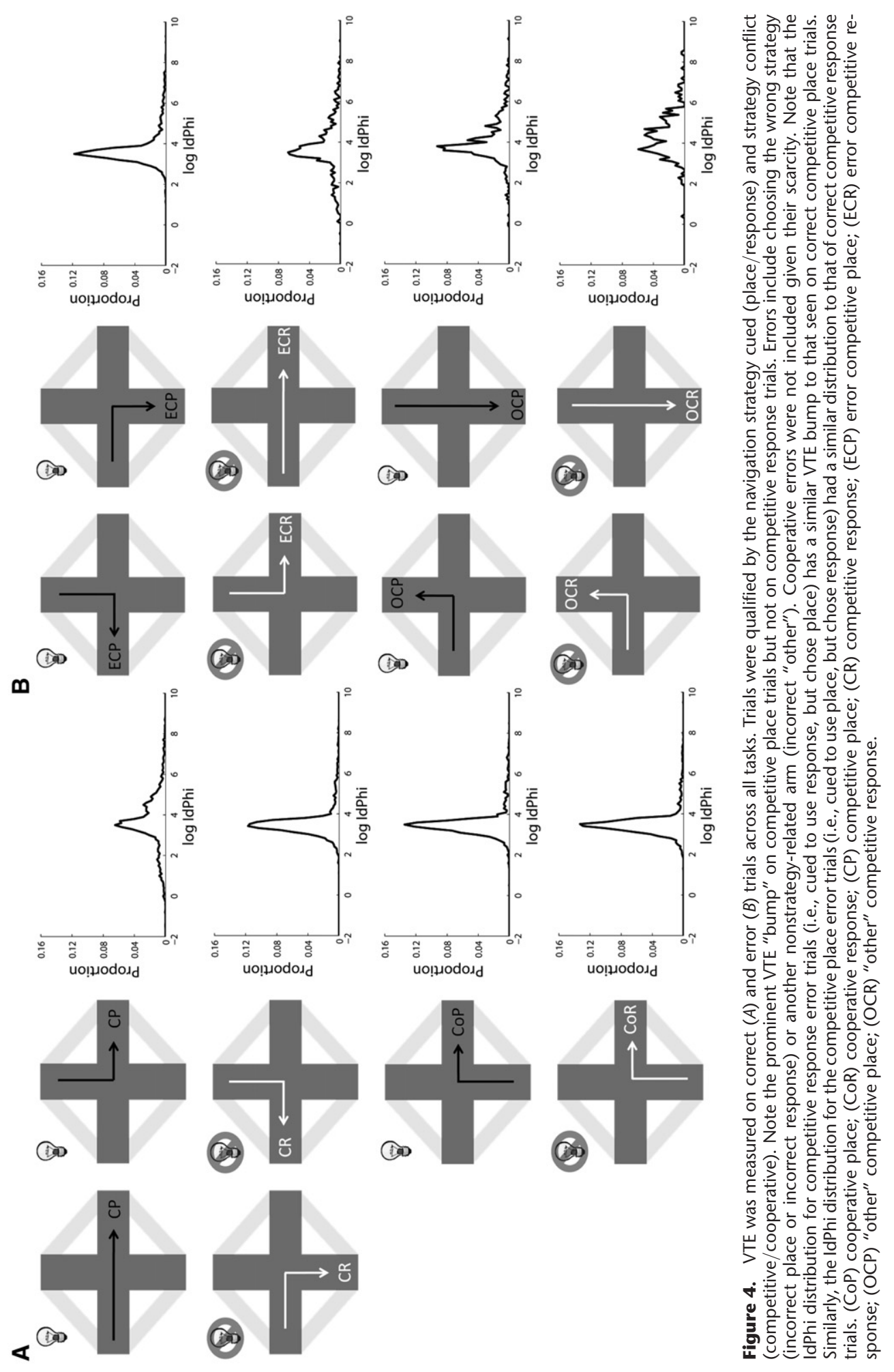



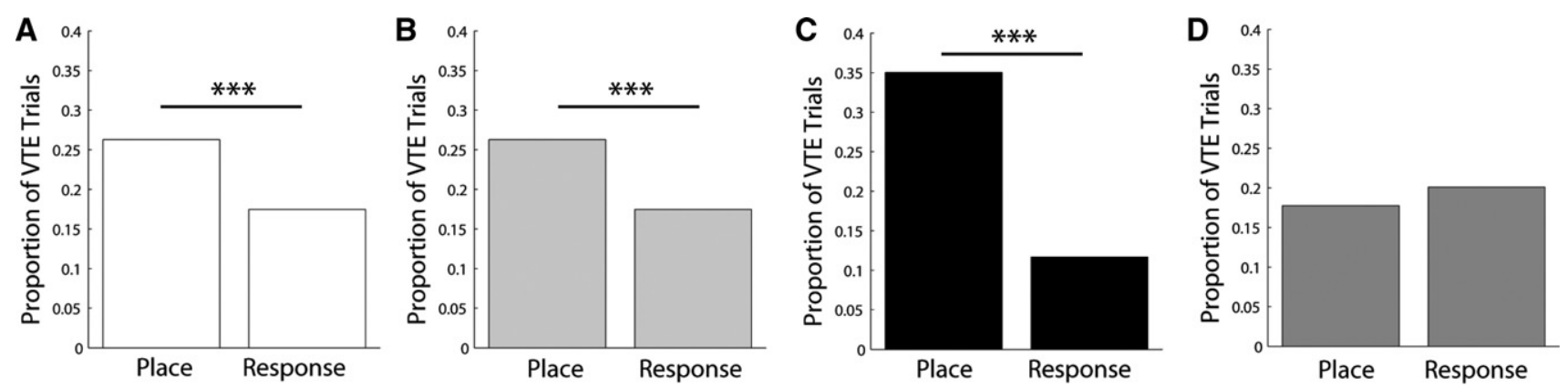

Figure 5. Proportion of place and response cued trials exhibiting VTE (qualified as log IdPhi $>4$ ). (A) Across all tasks, VTE was preferentially seen on place trials compared to response trials. VTE was significantly more likely on place trials on both the Fixed Task $(B)$ and the Novel Place Task $(C)$. (D) No significant differences were seen on the Novel Response Task. $\left.{ }^{* * *}\right) P<0.01$.

When error and correct trials were collapsed across the three tasks, VTE was greater on error trials than on correct trials $\left(\chi^{2}=\right.$ 248.1, $P<0$.0001) (Fig. 7A). Examining between tasks revealed a consistent pattern. VTE was more prominent on error trials than on correct trials on the Fixed Task $\left(\chi^{2}=48.2, P<0.0001\right)$ (Fig. 7B), Novel Place Task $\left(\chi^{2}=54.4, P<0.0001\right)$ (Fig. 7C), and Novel Response Task $\left(\chi^{2}=145.0, P<0.0001\right)$ (Fig. 7D). As expected, reaction time was also greater on error trials (Kruskal-Wallis; $\left.\chi^{2}=324.27, P<0.0001\right)$.

\section{Vicarious trial and error: Effects of error trial}

Across all tasks, VTE was greater on error trials. Therefore, we further examined VTE on error trials, this time matching it to each trial before an error trial (regardless of whether it was a correct or error trial) and after an error trial (regardless of whether it was a correct or error trial) (Fig. 8A). However, VTE was even greater on error trials than on the trial preceding it $\left(\chi^{2}=57.6, P<\right.$ 0.0001) (Fig. 8B). In contrast, VTE was greatest on trials following error trials $\left(\chi^{2}=21.1, P<0.0001\right)$ (Fig. $\left.8 \mathrm{~B}\right)$. Taken together, these results may suggest that the omission of reward put rats in a deliberative decision-making mode where they began looking for alternate available actions to obtain the goal. This could result in more VTE before reward on error trials and subsequent trials.

\section{Vicarious trial and error: Comparison across correct trial types}

Given that VTE was exhibited on place-cued trials and competitive trials, we assessed differences in VTE behavior across trial types (Fig. 8C). The four correct trial types were categorized by strategy cued (place/response) and strategy conflict (competitive/cooperative) (i.e., competitive place [ $n=3084$ ], competitive response $[n=3074]$, cooperative place $[n=2000]$, cooperative response $[n=1913])$. VTE was seen more on competitive place trials than any other trial type $\left(\chi^{2}=122.5-401.0\right.$, all $\left.P<0.0001\right)$ (Fig. $8 D)$. Additionally, VTE was seen more on competitive response trial types than on cooperative place or cooperative response trial types ( $\chi^{2}=9.0-9.2$, all $P<0.008$ ) (Fig. 8D). Interestingly, even when the animals were equally proficient on place and response strategies (i.e., the Fixed Task), VTE was still greater on competitive place trials than on competitive response trials $\left(\chi^{2}=12.9\right.$, $P<0.008$ ). Therefore, though VTE was consistently seen on error trials, it was also exhibited on correct trials, specifically during correct performance on place trials that required competition between both strategies.

\section{Discussion}

It has long been known that rats sometimes engage in vicarious trial and error (VTE) when making a choice (Muenzinger and Gentry 1931; Muenzinger 1938; Tolman 1939). The plus maze paradigm permitted the simultaneous examination of multiple VTE characteristics and expands upon our understanding of VTE behavior during deliberative decision-making. Given that hippocampal manipulations impair VTE (Hu and Amsel 1995; Blumenthal et al. 2011), we examined VTE on tasks with and without new hippocampal-dependent learning with the prediction that VTE would be greater during hippocampal-dependent learning. Consistent with this hypothesis, VTE was greater on the task requiring new hippocampal learning (Novel Place Task).
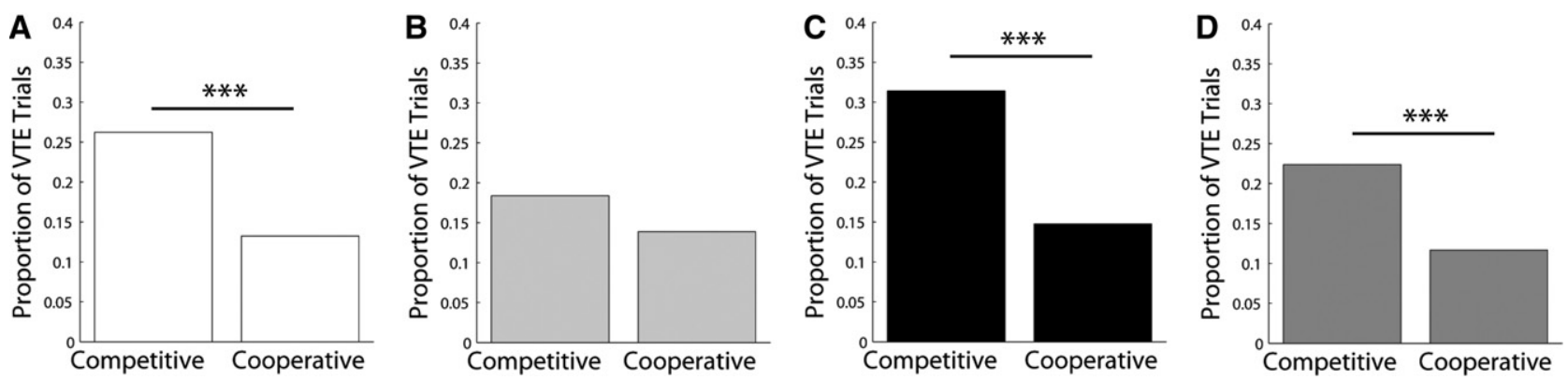

Figure 6. Proportion of competitive (place and response strategies indicated different goal locations) and cooperative (both place and response strategies indicated the same goal location) trials exhibiting VTE. (A) VTE was greater on competitive than on cooperative trials across tasks. (B) Similar, though nonsignificant, results were seen on the Fixed Task. Competitive trials exhibited more VTE than cooperative trials on the Novel Place Task (C) and the Novel Response Task $(D) .\left({ }^{* *}\right) P<0.01$. 

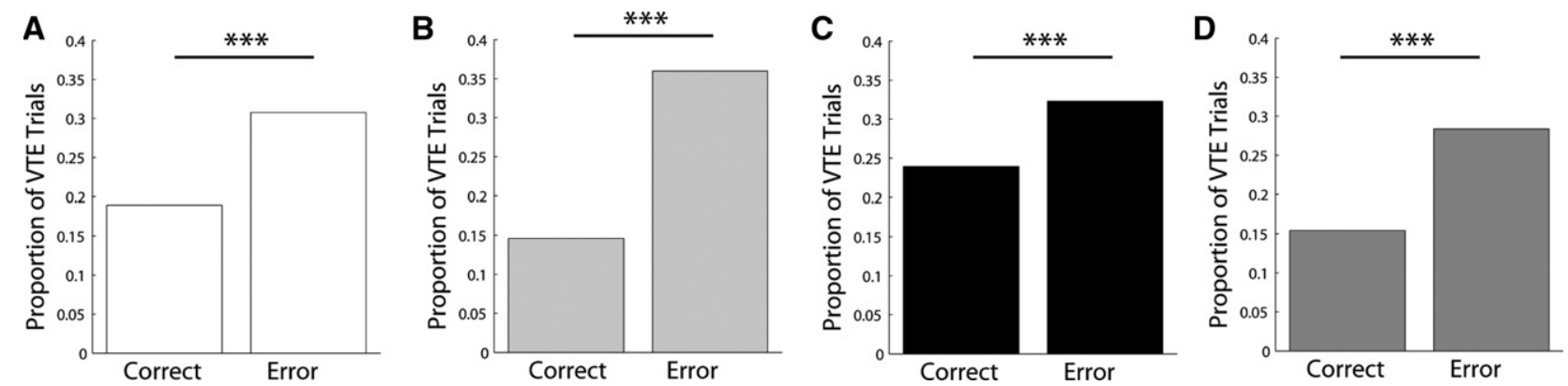

Figure 7. Proportion of correct and error trials exhibiting VTE. VTE was consistently greater on error trials than on correct trials across tasks $(A)$, on the Fixed Task $(B)$, on the Novel Place Task (C), and on the Novel Response Task $(D) .\left(^{* * *}\right) P<0.01$.

Similarly we measured VTE on hippocampal-dependent place trials and nonhippocampal-dependent response trials. As predicted, VTE was preferentially exhibited on place trials. VTE occurs early in learning and correlates with performance accuracy (Muenzinger and Gentry 1931; Muenzinger 1938; Tolman 1939). From these results, we predicted that VTE would be correlated with error trials. The results revealed that VTE was preferentially exhibited on error trials on all tasks. Additionally, measuring VTE before, on, and after error trials revealed that VTE was lowest before an error trial and greatest after an error trial. We suggest that an omission of a previously available reward engages the deliberative system, cuing rats to search for alternate actions to obtain a goal.

Johnson et al. (2012) suggest that VTE should occur when changing task demands are encountered in familiar environments. The Johnson et al. theory of directed exploration would predict a general increase in VTE during competitive trials, a supposition fully supported by our data. VTE was preferentially exhibited on competitive trials, particularly on competitive place trials. exhibited in rats during hippocampal place strategies. Across all tasks, VTE was greater when rats were cued to use a hippocampal-dependent place strategy than when cued to use a hippocampal-independent response strategy. In fact, the greatest VTE was found on the Novel Place Task, a spatial working memory task requiring the animals to learn a new place each day. Similarly, VTE was greater on place trials on the Fixed Task. In this task, rats were equally proficient on place and response strategies and made very few errors on both. Thus, even though both place and response trials were of equal training, the animals still exhibited more VTE when a hippocampus-based decision was required. Similarly, on correct trials, VTE was greatest on competitive place trials than on any other trial type (competitive response, cooperative place, and cooperative response). Interestingly, the rats exhibited VTE not only when correctly using a place strategy, but also when incorrectly using one (i.e., competitive response error trials [rat used a place strategy on the response trial]; see Fig. 4).

\section{VTE increases with hippocampal engagement}

During deliberative decision-making, animals use their knowledge of the world to internally evaluate the outcomes without actually experiencing them, a process believed to be supported by the hippocampus (Buckner and Carroll 2007; van der Meer et al. 2012). Likewise, when at a choice point, the rat may be considering the future consequences of its actions. Tolman (1939) predicted that rats imagine their prospective outcomes during VTE. As imagination and prediction are supported by the human hippocampus (Buckner and Carroll 2007; Hassabis et al. 2007), VTE in the rodent has been argued to be supported by hippocampal function ( $\mathrm{Hu}$ and Amsel 1995; Hu et al. 2006).

Lesion studies connect the hippocampus with VTE behavior (Hu and Amsel 1995) and the hippocampus internally represents potential paths during VTE behavior (Johnson and Redish 2007). However, to date VTE behavior has not been directly compared during hippocampal-dependent and hippocampal-independent strategies. The current data suggest that VTE is preferentially
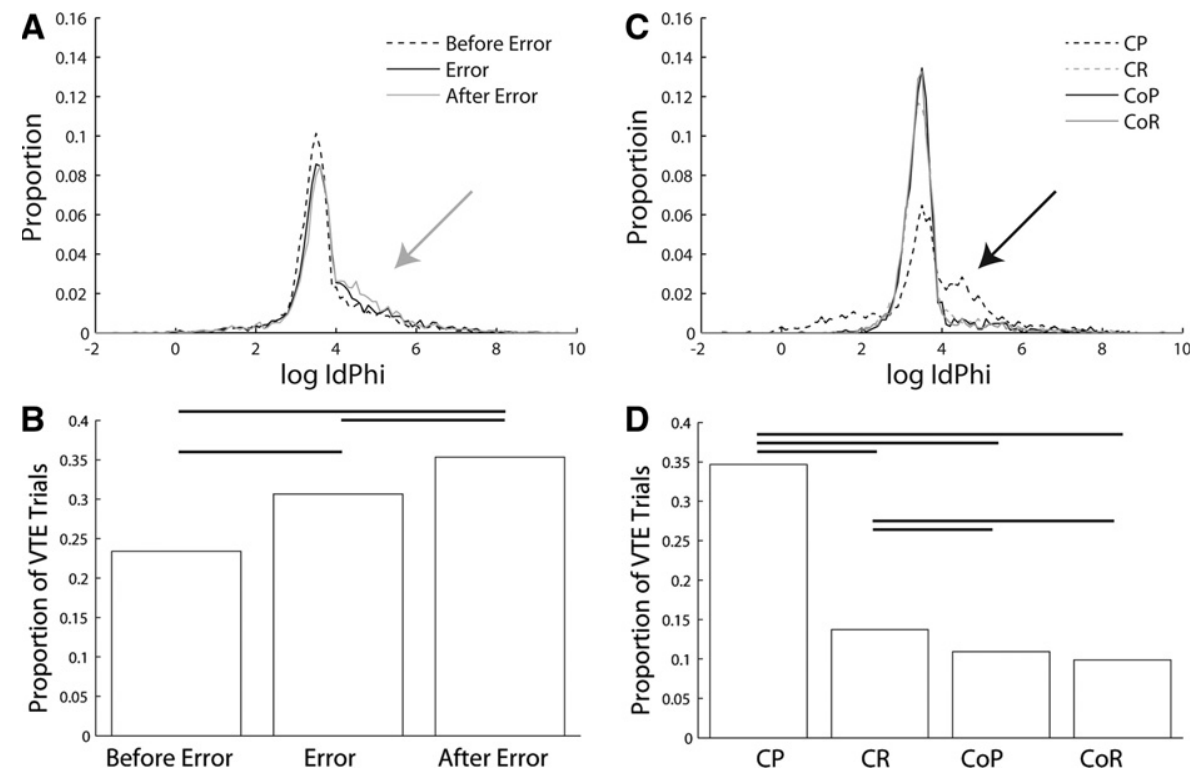

Figure 8. (A) VTE was examined before error trials, on error trials, and after error trials regardless of whether the trial preceding or following the error trial was a correct or error trial. Note the prominent bump (gray arrow) after error trials. (B) VTE was greater on an error trial than on the trial preceding it, but greatest after an error trial. (C) VTE was examined on all correct trial types across tasks. (CoP) cooperative place; (CoR) cooperative response; (CP) competitive place; (CR) competitive response. Note the prominent bump (black arrow) on competitive place trials. (D) VTE was greatest on competitive place correct trials than on any other trial type. VTE was greater on competitive response correct trials than on either cooperative trial type. (Black horizontal line) $P<0.008$. 
The current results are in agreement with previous studies that show increased VTE in rats utilizing hippocampal-dependent place strategies. Gardner et al. (2011) found that rats trained on a modified spatial maze task exhibited more VTE when they preferentially utilized a place strategy, but not in rats that preferentially utilized a response strategy during probe trials. Similarly, Hu et al. (2006) found that VTE behavior correlated with hippocampal activity, measured by cytochrome oxidase. Lesion and pharmacological manipulations further support the role of the hippocampus in VTE behaviors. Hippocampal lesions impaired VTE on a nonspatial discrimination task (Hu and Amsel 1995). Administrations of the NMDA antagonist CPP likewise disrupted VTE behaviors in rats trained on the spatial Multiple-T-Maze (Blumenthal et al. 2011).

While tasks engaging hippocampal processes result in more VTE, the dynamic between hippocampal involvement and VTE behavior is complex. First of all, hippocampal tasks such as place navigation also involve other structures (Whishaw et al. 1987; Packard et al. 1994; Devan and White 1999; Voss et al. 2011; Gruber and McDonald 2012). Although some studies have shown impairments in VTE and performance with hippocampal lesions (Hu and Amsel 1995), VTE has also been seen on hippocampusindependent tasks (Bett et al. 2012). The study by Bett and colleagues showed a more subtle relationship between the hippocampus and VTE behavior. Rats were trained to find food reward in one of four arms on a double-Y maze. After finding the food reward, the location of the reward was changed approximately every 10 trials. Sham animals appeared to show more VTE as they were exploring the maze to find the food reward. In contrast, once the food reward was found, the animals showed less VTE on successive trials. This was not the case for hippocampal-lesioned rats, which showed lower levels of VTE before finding the reward, but, unlike controls, did not show a decrease in VTE behavior on subsequent trials (Bett et al. 2012). Bett et al.'s results complement our study by suggesting that while VTE is greater when rats are engaged in strategies that are hippocampal-dependent, this does not necessitate that VTE is strictly a hippocampal process. Imaging studies in humans suggest that the prefrontal cortex may work together with the hippocampus during VTE-like behaviors (Voss et al. 2011).

\section{Task competition/conflict}

In the current study, rats were specifically trained to continuously switch between place and response strategies because it is well established that the hippocampal "place" system and the dorsalstriatal "motor-response" system are capable of compensating for one another during spatial navigation tasks (O'Keefe and Nadel 1978; Packard and McGaugh 1996).

These two navigational systems also compete with each other during decision-making. Previous studies (Schmidt et al. 2009; Jacobson et al. 2012) have shown that competitive trials were more difficult than cooperative trials. Competitive trials resulted in more errors; even at asymptomatic performance (Fixed Task), the few errors that did occur were predominantly on competitive trials. To further support the role of task conflict, when errors were made on competitive trials the rat would preferentially use the alternative navigation strategy. These studies demonstrate that these two systems are capable of interfering with each other during decision-making in spatial navigation tasks.

Theorists suggest that VTE occurs when changing task demands are encountered in familiar environments (Johnson et al. 2012), which is supported by the fact that rats trained on the Multiple-T-Maze show greater VTE after changes in reward contingency (Blumenthal et al. 2011). The Johnson et al. theory of directed exploration predicts a general increase in VTE during competitive trials, a supposition fully supported by our data. VTE is not only seen when rats are actively engaged in strategy switching (i.e., not an effect of set shifting), but on trials with conflict between different options (i.e., simultaneously holding different strategies "online"). Across all tasks, VTE behaviors were greater on trials that required place and response strategies to work competitively ("go to the place arm or make a right-hand turn") than on trials that allowed place and response trials to work cooperatively (going to the place arm and making a right-hand turn resulted in the same goal arm).

\section{Errors and trial accuracy suggest deliberative decision-making}

In the current paradigm we examined VTE on tasks where the rats were proficient at both strategies (Fixed Task) or required to learn a new place (Novel Place Task) and response (Novel Response Task) strategy. VTE was greatest on the Novel Place Task. Interestingly, no differences were seen between the task trained at asymptotic performance (Fixed Task) and the task with new hippocampal-independent learning (Novel Response Task). This suggests that hippocampal dependence is a mitigating factor above and beyond the general process of learning in VTE behavior.

The most consistent modulator of VTE across tasks was trial accuracy. VTE was greater on error trials than on correct trials on all three tasks. Interestingly, this effect was even seen on the Fixed Task. Thus even when a rat was overtrained and made few daily errors, those trials in which it was confused (i.e., made errors) were characterized by increased VTE.

Less expected was the finding that after making an error the animal showed high VTE on the next trial. When animals make an error they must repeat the trial type until successful (see Materials and Methods). Given that the animal received feedback regarding the correct choice, a repeat of the trial should be easier than the initial attempt. The fact that following an error the animal showed more VTE at the choice point supports the hypothesis that VTE behaviorally reflects deliberative decision-making. Normally when the rats became proficient at the task they paused and hesitated less at the choice point. The current results revealed that making an error may switch an animal from a more "automatic" to a more "reflective" mode, at least for the following trial. In other words, it was as though after an error trial the rats became uncertain and wanted to make sure they got it right the next time. This would suggest a switch to deliberative decision-making in response to errors (van der Meer et al. 2012).

\section{Summary}

When deciding what, how, and when to do something, different decision-making strategies are available. VTE is believed to behaviorally reflect the engagement of the deliberative decision-making system. Deliberative decision-making encompasses searching, predicting, and evaluating outcomes (van der Meer and Redish 2010). Similarly, when a rat pauses and orients at the choice point it is believed to be holding the information online and evaluating outcomes before experiencing them (Johnson and Redish 2007; van der Meer and Redish 2009). Deliberative decision-making is computationally slow, as VTE is behaviorally slow. Deliberative decision-making allows for flexible behavior; similarly, during VTE animals are scanning/evaluating their options, and VTE behavior decreases with automation (Johnson and Redish 2007; van der Meer and Redish 2009).

The current study sheds light on what pushes a rat into a deliberative decision-making mode (vs. other decision-making processes) by evaluating what behavioral paradigm parameters engaged a rat in deliberative decision-making behavior, as 
measured by VTE. Several behavioral paradigm parameters promoted deliberative decision-making. Engaging the hippocampus, by introducing novel place learning or even using just a hippocampal place strategy, produced increased VTE behavior. Additionally, VTE was consistently exhibited on error trials. Thereby, when at the choice point and uncertain about what to do, the rat paused and purportedly evaluated its potential outcomes; though computationally slow, this behavior was exhibited over automatically running through the choice point. Interestingly, VTE behavior was increased not only on error trials, but was potentiated even more on the following trial. This suggests that the state of uncertainty seen at the choice point during error trials potentially pushes animals to preferentially use the deliberative decision-making system. Finally, VTE was seen on trials where the rats needed to be flexible to changing strategy demands. VTE was preferentially exhibited on the more difficult competitive trials, trials that required actively choosing between the hippocampal-dependent place strategy and hippocampal-independent response strategy.

\section{Materials and Methods}

\section{Subjects}

Eleven male Fisher 344 rats (Harlan, IL) were used in this study. All of the rats were housed in a vivarium maintained at $72.3^{\circ} \mathrm{C}$ and kept on an 8:00/8:00 light-dark cycle. The rats were kept individually in clear Plexiglas cages $(46 \mathrm{~cm} \times 20 \mathrm{~cm} \times 23 \mathrm{~cm})$ with pine bedding and a free water bottle. The rats were maintained at $85 \%$ of their ad lib weight during the experiment. All procedures were performed in accordance with the University of Connecticut's Institutional Animal Care and Use Committee.

\section{Apparatus}

A black Plexiglas runway $(120.7 \mathrm{~cm} \times 10.2 \mathrm{~cm})$ was used for pretraining. A modified version of the plus maze was used for training. The plus maze was constructed of black Plexiglas $(112.4 \mathrm{~cm}$ long, $10.8 \mathrm{~cm}$ wide, $15.9 \mathrm{~cm}$ off the table). Four black Plexiglas runways were constructed to form a perimeter around the plus maze.

\section{Pretraining}

Rats were trained to run back and forth on a linear runway for chocolate sprinkle rewards once a day, daily for $10 \mathrm{~d}$. The daily training sessions continued for up to $10 \mathrm{~min}$ on the maze or until the rat reached the criterion of 10 trials in 5 min.

\section{Behavioral procedure}

Each training session consisted of 32 correct trials or 20 min, whichever came first $(n=7)$ and 40 correct trials or $30 \mathrm{~min}(n=$ 4). Training continued until a criterion of two consecutive days at $80 \%$ correct (unless otherwise specified) was reached, after which the rat proceeded to the next training paradigm.

\section{Experiment 1: Fixed Place and Response Task ("Fixed Task")} Training procedures were as described in Schmidt et al. (2009) and Jacobson et al. (2012). Briefly, first rats were trained to perform a response task ("right-hand turn"). Each trial ended after the rat entered a maze arm, whether or not a correct choice was made. Perimeter runways connecting the arms were raised, providing a path to the next start location (if an error was made, the rat was returned to the original start arm to repeat the trial until successful). Once the rat reached the criterion for response training, the place strategy was introduced. During place and response block training, the sessions were broken up roughly into half place/ half response blocks (about 16 trials each), such that animals switched strategies one or two times. Again, during the response block, rats were rewarded for making a right-hand turn on the maze. During the place block trials (spatial reference memory task), rats were rewarded for going to the same "place" (east arm) regardless of the start arm. The place trials were differentiated from the response trials by a flashing light situated near the ceiling, which illuminated the room and remained on for the duration of the place trials. Training continued until each rat reached the criterion (two consecutive days at $80 \%$ correct for each trial type), after which the rat commenced training on the fixed place and response training (Fig. 1A). During fixed place and response training, each rat was given a similar number of place and response trials within a session. However, the place and response trials were interwoven with no more than three consecutive trials of one trial type. Once rats reached the criteria, they were trained 2-3 times per week until surgery and given a week to recover. When recovered, the rats were retrained on the Fixed Place and Response Task until they reached the criteria, and then commenced training on the Novel Place and Fixed Response Task.

\section{Experiment 2: Novel Place and Fixed Response Task ("Novel Place Task")}

Rats were trained to run to a new place goal arm each day during the place trials, while continuing to make a right-hand turn during the response trials (Fig. 1A). Again, place and response trials were interwoven with no more than three consecutive trials of one trial type. At the start of each training session, the rat was placed at the end of the designated goal arm, while blocked from the rest of the maze, for $\sim 30 \mathrm{sec}$ with the flashing light on and a full cup of chocolate sprinkle reward. After the exposure, the perimeter arms were raised and the rat was guided to the start arm. The correct place arm was pseudo-randomly assigned every day (north, south, east, west). Given the difficulty of the task, the place task criterion was reduced to $60 \%$ correct, while the response criterion was maintained at $80 \%$ correct. Rats were trained until a criterion was reached, plus three nonconsecutive criteria days.

\section{Experiment 3: Novel Response and Fixed Place Task ("Novel Response Task")}

Rats were trained to make a new motor response, a left-hand turn (Fig. 1A) during the response trials, while continuing to go to the original place arm (east arm) during the place trials. Place and response trials were interwoven with no more than three consecutive trials of one trial type. Training continued until each rat reached the criteria (two consecutive days at 80\% correct for both trial types) and three subsequent criteria days.

\section{Maze performance analysis}

The configuration of the plus maze allowed for a trial analysis based on the type of learning strategy that could be used (Figs. $1 \mathrm{~B}, 4 \mathrm{~A})$. On a competitive arm, the rat could use a place strategy, which would lead to the east arm, or a response strategy and make a right-hand turn (i.e., both strategies indicate different arms). Additionally, on a competitive trial the rat could go to the "other" arm which was not indicated by either the place or response strategy. On a cooperative arm, the rat could use a place strategy which would lead to the east arm or a response strategy and make a righthand turn ending on the east arm (i.e., both strategies indicate the same arm) (Schmidt et al. 2009; Jacobson et al. 2012). Therefore, each error type was qualified based on the strategy cued (place/ response) and strategy conflict (competitive/cooperative); for example, a competitive place error is when a rat is on a competitive arm but incorrectly uses a response strategy, a competitive place "other" error is when a rat is on a competitive arm but goes to an arm not indicated by either the place or response strategy, and a cooperative place error is when a rat is on a cooperative arm but goes to the north or west arm (Fig. 4B).

\section{VTE measurement with IdPhi}

An overhead camera sampling at $30 \mathrm{~Hz}$ tracked the position of the rat from an LED-mounted headstage. Illuminated pixels that 
exceeded a user-defined threshold were digitally timestamped and recorded using a Cheetah data acquisition system. Recorded data were deinterlaced with linear interpolation between consecutive frames to smooth the digitized image. Custom analysis programs were written using Matlab.

The $x$ and $y$ components of velocity were computed using an adaptive windowing algorithm (Janabi-Sharifi et al. 2000). Change in head angle Phi was computed using the arctangent of these components, and unwrapped to prevent circular transitions. Head acceleration $d P h i$ was then computed with the adaptive windowing algorithm (Janabi-Sharifi et al. 2000). The absolute value $|d P h i|$ was integrated in time to produce IdPhi. Log IdPhi values greater than 4.0 were qualified as a VTE event.

Position samples crossing the center of the plus maze were used for IdPhi analysis. Analysis began at the first sample that crossed the center of the plus maze and ended when the rat entered any of the four arms of the plus maze. Due to technical errors in video tracking, one rat was excluded from analysis.

\section{Statistics}

A $2 \times 3$ ANOVA was used to test for main effects and interactions. A $\chi^{2}$ was used to test significance between proportions of trials exhibiting VTE. Significance values were corrected for multiple comparisons across tasks and trial types.

\section{Acknowledgments}

We thank Melissa Argraves, Dr. Elizabeth Ciurylo, Jia Li Lu, Emma Zhang, Benjamin Gruenbaum, and Joshua Fairchild for their assistance with behavioral training. This research was supported by the University of Connecticut Research Foundation (E.J.M. and B.S.) and the Human Frontiers Science Program Project Grant HFSPRGP0039/2010 (A.D.R., B.S., and A.P.).

\section{References}

Bett D, Allison E, Murdoch LH, Kaefer K, Wood ER, Dudchenko PA. 2012. The neural substrates of deliberative decision making: Contrasting effects of hippocampus lesions on performance and vicarious trial-and-error behavior in a spatial memory task and a visual discrimination task. Front Behav Neurosci 6. doi: 10.3389/ fnbeh.2012.00070.

Blumenthal A, Steiner A, Seeland K, Redish AD. 2011. Effects of pharmacological manipulations of NMDA receptors on deliberation in the Multiple T task. Neurobiol Learn Mem 95: 376-384.

Buckner RL, Carroll DC. 2007. Self projection and the brain. Trends Cogn Sci 11: $49-57$.

Devan BD, White NM. 1999. Parallel information processing in the dorsal striatum: Relation to hippocampal function. J Neurosci 19: 2789-2798.

Gardner RS, Fleming SE, Uttaro MR, Suarez DF, Adams AG, Ascoli GA, Dumas TC. 2011. Sequential task demands affect place and response strategy recruitment on a modified plus-maze. In 2011 Neuroscience meeting planner, abstract program no 611.04. Society for Neuroscience, Washington, DC.

Gruber AJ, McDonald RJ. 2012. Context, emotion, and the strategic pursuit of goals: Interactions among multiple brain systems controlling motivated behavior. Front Behav Neurosci 6. doi: 10.3389/fnbeh. 2012.00050.
Hassabis D, Kumaran D, Vann SD, Maguire EA. 2007. Patients with hippocampal amnesia cannot imagine new experiences. Proc Natl Acad Sci 104: $1726-1731$.

Hu D, Amsel A. 1995. A simple test of the vicarious trial-and-error hypothesis of hippocampal function. Proc Natl Acad Sci 92: 5506-5509.

$\mathrm{Hu}$ D, Xu X, Gonzalez-Lima F. 2006. Vicarious trial-and-error behavior and hippocampal cytochrome oxidase activity during Y-maze discrimination learning in the rat. Int J Neurosci 116: 265-280.

Jacobson TK, Gruenbaum BF, Markus EJ. 2012. Extensive training and hippocampus or striatum lesions: Effect on place and response strategies. Physiol Behav 105: 645-652.

Janabi-Sharifi F, Hayward V, Chen CSJ. 2000. Discrete-time adaptive windowing for velocity estimation. IEEE Trans Control Syst Tech 8: $1003-1009$

Johnson A, Redish AD. 2007. Neural ensembles in CA3 transiently encode paths forward of the animal at a decision point. J Neurosci 27: $12176-12189$.

Johnson A, Varberg Z, Benhardus J, Maahs A, Schrater P. 2012. The hippocampus and exploration: Dynamically evolving behavior and neural representations. Front Hum Neurosci 6: 216. doi: 10.3389/ fnhum.2012.00216.

Muenzinger KF. 1938. Vicarious trial and error at a point of choice. I. A general survey of its relation to learning efficiency. J Genet Psychol 53: $75-86$.

Muenzinger KF, Gentry E. 1931. Tone discrimination in white rats. J Comp Psychol 12: 195-206.

O'Keefe J, Nadel L. 1978. The hippocampus as a cognitive map. Clarendon Press, Oxford, UK.

Packard MG. 1999. Glutamate infused posttraining into the hippocampus or caudate-putamen differentially strengthens place and response learning. Proc Natl Acad Sci 96: 12881-12886.

Packard MG, McGaugh JL. 1996. Inactivation of hippocampus or caudate nucleus with lidocaine differentially affects expression of place and response learning. Neurobiol Learn Mem 65: 65-72.

Packard MG, Cahill L, McGaugh JL. 1994. Amygdala modulation of hippocampal-dependent and caudate nucleus-dependent memory. Proc Natl Acad Sci 30: 8477-8481.

Papale AE, Stott JJ, Powell NJ, Regier PS, Redish AD. 2012. Interactions between deliberation and delay-discounting in rats. Cogn Affect Behav Neurosci 12: 513-526.

Redish AD. 1999. Beyond the cognitive map: From place cells to episodic memory. MIT Press, Cambridge, MA.

Schmidt B, Jacobson TK, Markus EJ. 2009. Hippocampal and striatal dependent navigation: Sex differences are limited to acquisition. Horm Behav 56: 199-205.

Tolman EC. 1938. The determiners of behavior at a choice point. Psychol Rev 45: $1-41$.

Tolman EC. 1939. Prediction of vicarious trial and error by means of the schematic sowbug. Psychol Rev 46: 318-336.

van der Meer MAA, Redish AD. 2009. Covert expectation-of-reward in rat ventral striatum at decision points. Front Integr Neurosci 3: 1-15.

van der Meer MAA, Redish AD. 2010. Triple dissociation of information processing in dorsal striatum, ventral striatum, and hippocampus on a learned spatial decision task. Neuron 67: 25-32.

van der Meer MAA, Kurth-Nelson Z, Redish AD. 2012. Information processing in decision-making systems. Neuroscientist 18: 342-359.

Voss JL, Warren DE, Gonsalves BD, Federmeier KD, Tranel D, Cohen NJ. 2011. Spontaneous revisitation during visual exploration as a link among strategic behavior, learning, and the hippocampus. Proc Natl Acad Sci 108: E402-E409.

Whishaw IQ, Mittlema G, Bunch ST, Dunnet SB. 1987. Impairments in the acquisition, retention and selection of spatial navigation strategies after medial caudate-putamen lesions in rats. Behav Brain Res 24: $125-138$.

Received September 24, 2012; accepted in revised form December 20, 2012. 


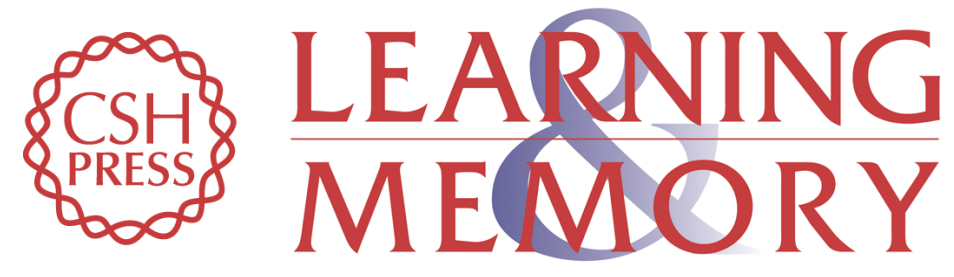

\section{Conflict between place and response navigation strategies: Effects on vicarious trial and error (VTE) behaviors}

Brandy Schmidt, Andrew Papale, A. David Redish, et al.

Learn. Mem. 2013, 20:

Access the most recent version at doi:10.1101/Im.028753.112

References This article cites 25 articles, 6 of which can be accessed free at:

http://learnmem.cshlp.org/content/20/3/130.full.html\#ref-list-1

License

Email Alerting Receive free email alerts when new articles cite this article - sign up in the box at the Service top right corner of the article or click here. 